\title{
Association between Brachial-Ankle Arterial Stiffness and Cerebral Small Vessel Disease Load in Patients with Acute Non- Cardioembolic Ischemic Stroke
}

\author{
MeyungKug Kim, Bong Goo Yoo* \\ Department of Neurology, Kosin University College of Medicine, Busan, Rep. of Korea
}

\begin{abstract}
*Corresponding Author: Bong Goo Yoo, Department of Neurology, Kosin University College of Me dicine, Busan, Rep. of Korea, Email: munguss@naver.com
\end{abstract}

\begin{abstract}
Background: Increased arterial stiffness may lead to vessel damage and atherosclerosis, with the former occurring more frequently in the microvessels of end organs. We investigated the association between arterial stiffness, measured via brachial-ankle pulse wave velocity (baPWV), and cerebral small vessel disease (CSVD), visualized on magnetic resonance imaging (MRI) as white matter hyperintensities (WMHs), lacunar infarctions, and cerebral microbleeds $(C M B s)$.
\end{abstract}

Methods: This study included 88 patients with ischemic stroke who were diagnosed with non-cardioembolic acute cerebral infarction. All patients underwent baPWV and brain MRI. The number of CMBs and lacunar infarctions was evaluated. The locations of CMBs and lacunar infarctions were divided into infratentorial, lobar, and deep regions, including the basal ganglia, thalamus, and internal capsule. Moreover, WMHs were divided into deep and periventricular regions, and their severity was assessed.

Results: Increased baPWV was associated with lacunar infarctions and WMHs ( $p<0.05)$, but not with CMBs. On multivariate analysis, the association between baPWV and lacunar infarctions in the deep regions was stronger than that between baPWV and the other imaging markers of CSVD $(p<0.01)$.

Conclusions: These findings suggest that increased arterial stiffness may be associated with CSVD severity. Moreover, lacunar infarctions in the deep regions, such as the basal ganglia and thalamus, were related to arterial stiffness.

Keywords: Ankle brachial index, Cerebral small vessel diseases, Magnetic resonance imaging, White matter, Lacunar infarcts

\section{INTRODUCTION}

The arterial pulse wave velocity (PWV), a known independent predictor of cardiovascular disease, is widely used in clinical settings to determine changes in blood vessels caused by atherosclerosis. $^{1,2}$ PWV increases as arteriosclerosis worsens, and increased arterial stiffness exacerbates vascular damage and atherosclerosis. ${ }^{3,4}$ It mainly affects the small vessels of the end organ.The brachial-ankle pulse wave velocity (baPWV), most commonly used for its simple and reproducible measurements,reflects thecentral and peripheral arterial stiffness. ${ }^{2}$

Cerebral small vessel disease (CSVD) is a major form of ischemic stroke. Although its pathogenesis is unclear, arterial stiffness associated with high blood pressure plays a major role in its pathophysiology. ${ }^{5}$

The markers of CSVD include lacunar infarction, white matter hyperintensity (WMH), cerebral microbleed (CMB), and dilated perivascular space (PVS). ${ }^{5}$ Although increased arterial stiffness has been linked to lacunar infarction, $\mathrm{WMH}$, and $\mathrm{CMB}$, previous studies have reported inconsistent results. ${ }^{5-8}$

To understand their relation, we compared the severity of imaging markers of CSVD, such as WMH, lacunar infarction, and CMB, visualized using brain magnetic resonance imaging (MRI), and arterial stiffness, measured using baPWV, in the patients with acute ischemic stroke. 


\section{MATERIALS AND METHODS}

\subsection{Materials}

Between March 2018and July 2020, 628 consecutive patients with acute ischemic stroke who were admitted within 7 days of symptom onset were enrolled.All patients were hospitalized with neurological symptoms and were diagnosed with cerebral infarction on brain MRI.A total of 193 patients with embolic cerebral infarction of cardiac origin, such as atrial fibrillation, a trial flutter, patent foramenovale, artificial heart valve, mitral valve stenosis, thrombus in the left atrium, or atrial appendage, were excluded because of the difficulty in measuring baPWV. A total of 233 patients who did not have their baPWV measured and 62 patients with cardiac disorders, such as congestive heart failure, myocardial infarction within 6 months, and dilated cardiomyopathy, were also excluded. We further excluded 52 patients who had poor quality MRI data and missing data in medical records. Atotal of 88 patients werefinally enrolled in the study.

\subsection{Methods}

The patients' clinical data were obtained through medical records and interviews, including their medical history, past history, and risk factors for cerebrovascular disease. Blood tests, electro cardiogram, and echocardiography were also conducted.Hypertension was defined as a systolic blood pressure of $>140 \mathrm{mmHg}$ or a diastolic blood pressure of $>90 \mathrm{mmHg}$ after the acute period of stroke and current use of antihypertensive medication, regardless of the blood pressure. Diabetes mellitus was defined as diabetes treatment, fasting glucose level $>126$ $\mathrm{mg} / \mathrm{dL}$ after hospitalization, oral glucose tolerance test result $>200 \mathrm{mg} / \mathrm{dL}$ at 2 hours, or hemoglobin A1c level >6.5 \%A1c. Hyperlipidemia was defined as fasting total cholesterol level >240 mg/dL, low-density lipoprotein level $>160 \mathrm{mg} / \mathrm{dL}$, or use of lipidlowering medication.

Arterial stiffness and the severity of CSVD in the brain were evaluated using baPWV and brain MRI, respectively.After the patient had rested in a supine position for at least $5 \mathrm{~min}$, baPWV was measured using a volumeplethysmography apparatus (VP-1000; Collin, Co., Ltd., Komaki, Japan), which simultaneously records the PWV, arterial blood pressure, electrocardiogram, and heart sounds at the upper arms and ankles on both sides. The
baPWV was calculated using time-phase analysis between the right arm andvolume waveforms at both ankles. The distance between the rightbrachium and the ankle was estimated accordingto the body height. The average baPWV values obtained from both sides were then used forfurther analysis.The patients were then divided into tertiles depending on their baPWV; there were 29 patients with a baPWV of $<1,699 \mathrm{~cm} / \mathrm{s}, 30$ patients with a baPWV of $1,699-2,023 \mathrm{~cm} / \mathrm{s}$, and 29 patients with a baPWV of $>2,023 \mathrm{~cm} / \mathrm{s}$.

Axial T1- and T2-weighted, fluid-attenuated inversion recovery, and gradient-echo $\mathrm{T} 2 *_{-}$ weighted images were collected using a 1.5-T MRI (GE HealthCare, Waukesha, WI). The number of CMBs and lacunar infarctions was identified usingthe gradient-echo and T1/T2weighted images, respectively. Using the Microbleed Anatomical Rating Scale, the locations of theCMBs and lacunar infarctions wereclassified into infratentorial, lobar, anddeep regions. ${ }^{9} \mathrm{WMHs}$ were evaluated using theClinical Research for Dementia of SouthKoreavisual rating score. ${ }^{10}$ Specifically, periventricular WMHs were evaluated on P1 (cap and band, <5 mm), P2 (cap or band, 5-10 $\mathrm{mm}$ ), and P3 (cap or band, >10 mm).Moreover, deep WMHs wereevaluated as D1 $(<10 \mathrm{~mm})$, D2 (10-25 mm), and D3 (>25 mm) based on the maximum diameter. WMHs werecategorized asperiventricular, deep, and mixed based on their location andwere graded as minimal, moderate, and severe $(1,2$, and 3 points, respectively). The assessments wereperformed by a neurologist.

\subsection{Statistics}

For statistical analyses, patients were divided into the tertiles according to baPWV. Continuous variables were analyzed using the Kruskal-Wallis test, and categorical variables were analyzed using linear-by-linear association.Multivariate analysis was performed to determine the variables associated with baPWV. First, we adjusted for age, sex, and systolic blood pressure (model 1). In model 2, we additionally adjusted for heart rate, hemoglobin level, erythrocyte sedimentation rate, and glomerular filtration rate. Statistical Package for the Social Sciences Version 19.0 (SPSS Inc., Chicago, IL, USA) was used.A pvalue of $<0.05$ was considered significant. 


\section{RESULTS}

\subsection{Clinical Characteristics According to baPWV Differences}

Among the three groups divided by tertiles, sex, age, systolic and diastolic blood pressure, pulse pressure, heart rate, hemoglobin level, erythrocyte sedimentation rate, and glomerular filtration rate were significantly different $(p<0.05)$. However, factors such as presence of hypertension, diabetes mellitus, and hyperlipidemia; smoking; alcohol consumption; prior history of stroke or coronary heart disease; serum creatinine level; fasting glucose level; total cholesterol level; and body mass indexwere not different among the groups (Table 1).

Table1: Baseline demographics and characteristics of patients with acute ischemic stroke according to pulse wave velocity

\begin{tabular}{|c|c|c|c|c|}
\hline & $\begin{array}{l}\text { baPWV } \\
(<1699 \\
(\mathrm{n}=29)\end{array}$ & $\begin{array}{l}\text { baPWV } \\
(1699-2023 \\
\mathrm{cm} / \mathrm{s})(\mathrm{n}=30)\end{array}$ & $\begin{array}{l}\mathrm{baPWV} \\
(>2023 \mathrm{~cm} / \mathrm{s}) \\
(\mathrm{n}=29)\end{array}$ & $p$-value \\
\hline Sex (Male/Female) & $23 / 6$ & $21 / 9$ & $14 / 15$ & 0.038 \\
\hline Age (years) & $58.8 \pm 10.2$ & $70.6 \pm 9.7$ & $70.6 \pm 8.5$ & $<0.001$ \\
\hline Hypertension, n (\%) & $13(44.8)$ & $14(46.7)$ & $15(51.7)$ & 0.601 \\
\hline Diabetes mellitus, n (\%) & $3(10.3)$ & $10(33.3)$ & $9(31.0)$ & 0.070 \\
\hline Hyperlipidemia, n (\%) & $5(17.2)$ & $4(13.3)$ & $4(13.8)$ & 0.713 \\
\hline Current Smoking, $\mathrm{n}(\%)$ & $11(37.9)$ & $9(30.0)$ & $6(20.7)$ & 0.152 \\
\hline Alcohol, n (\%) & $11(37.9)$ & $13(43.3)$ & $10(34.5)$ & 0.789 \\
\hline Prior stroke, $\mathrm{n}(\%)$ & $4(13.8)$ & $3(10.0)$ & $5(17.2)$ & 0.704 \\
\hline Coronary artery disease, $\mathrm{n}(\%)$ & $2(6.9)$ & $2(6.7)$ & $2(6.9)$ & 0.971 \\
\hline Systolic BP (mmHg) & $133.3 \pm 12.4$ & $146.0 \pm 22.4$ & $162.8 \pm 26.4$ & $<0.001$ \\
\hline Pulse pressure $(\mathrm{mmHg})$ & $51.9 \pm 9.3$ & $62.1 \pm 13.4$ & $66.9 \pm 15.5$ & $<0.001$ \\
\hline Diastolic BP (mmHg) & $81.4 \pm 8.4$ & $87.0 \pm 22.9$ & $91.8 \pm 10.6$ & 0.004 \\
\hline Heart rate (beats/min) & $65.0 \pm 8.5$ & $63.3 \pm 15.3$ & $72.5 \pm 12.1$ & 0.021 \\
\hline Hemoglobin $(\mathrm{g} / \mathrm{dL})$ & $14.3 \pm 1.4$ & $13.4 \pm 1.7$ & $13.1 \pm 1.2$ & 0.017 \\
\hline $\operatorname{ESR}(\mathrm{mm} / \mathrm{hr})$ & $13.3 \pm 9.4$ & $20.8 \pm 21.7$ & $28.2 \pm 24.6$ & 0.017 \\
\hline Creatine $(\mathrm{mg} / \mathrm{dL})$ & $0.8 \pm 0.2$ & $1.0 \pm 1.1$ & $1.01 \pm 0.7$ & 0.510 \\
\hline GFR $(\mathrm{mL} / \mathrm{min})$ & $101.8 \pm 23.7$ & $98.5 \pm 23.3$ & $80.8 \pm 37.2$ & 0.015 \\
\hline Fasting blood sugar $(\mathrm{mg} / \mathrm{dL})$ & $105.7 \pm 33.3$ & $125.3 \pm 102.8$ & $108.4 \pm 21.6$ & 0.282 \\
\hline Total cholesterol (mg/dL) & $193.0 \pm 45.4$ & $174.1 \pm 63.1$ & $192.5 \pm 42.0$ & 0.412 \\
\hline Body mass index $\left(\mathrm{kg} / \mathrm{m}^{2}\right)$ & $23.6 \pm 2.0$ & $23.9 \pm 2.7$ & $22.9 \pm 2.8$ & 0.645 \\
\hline
\end{tabular}

Values are presented as mean \pm standard deviation or number (\%).

baPWV, brachial-ankle pulse wave velocity; BP, blood pressure; ESR, erythrocyte sedimentation rate; GFR, glomerular filtration rate.

\subsection{Relation between baPWV and Brain Imaging Markers of CSVD}

There were no significant differences in the degree and location of CMBsamong the three groups divided by tertiles(Table 2). There were significant differences in the total number of lacunar infarctionsand deep lacunar infarctions $(p<0.01)$.Further, there was no difference in the number of lacunar infarctions in the infratentorial and lobar areas (Table 2). WMHs were significantly more severe in the higher baPWV group, both in the deep and periventricular regions $(p<0.05)$. There was also a significant difference in the total $\mathrm{WMH}$ grade ( $\mathrm{p}=0.042$; Table 2). Therefore, baPWV was associated with WMHs and the severity of lacunar infarction, but not with CMBs.

\subsection{Multivariate Analysis of the Association between CSVD and baPWV}

Multivariate analysis was performed by adjusting forvariable factors. In model 1, baPWV was significantly associated with the total number of lacunar infarctions, deep and lobarlacunar infarctions, and periventricular WMHs ( $<<0.05$; Table 3). However, in model 2, which adjusted formore variables, only deeplacunar infarction was significantly associated with baPWV ( $\mathrm{p}<0.01$; Table 3$)$. 
Association between Brachial-Ankle Arterial Stiffness and Cerebral Small Vessel Disease Load in Patients with Acute Non-Cardioembolic Ischemic Stroke

Table2: Imaging markers of small vessel disease according to tertiles between brachial-ankle pulse wave velocity

\begin{tabular}{|l|l|l|l|l|}
\hline & $\begin{array}{l}\text { baPWV } \\
(<1699 \mathrm{~cm} / \mathrm{s}) \\
(\mathrm{n}=29)\end{array}$ & $\begin{array}{l}\text { baPWV } \\
(1699-2023 \mathrm{~cm} / \mathrm{s}) \\
(\mathrm{n}=30)\end{array}$ & $\begin{array}{l}\text { baPWV } \\
(>2023 \mathrm{~cm} / \mathrm{s}) \\
(\mathrm{n}=29)\end{array}$ & $p$-value \\
\hline Cerebral microbleeds & & & $1.24 \pm 2.53$ & 0.488 \\
\hline Total & $1.24 \pm 4.62$ & $1.50 \pm 4.02$ & $0.28 \pm 0.92$ & 0.531 \\
\hline Infratentorial & $0.14 \pm 0.58$ & $0.23 \pm 0.57$ & $0.69 \pm 1.56$ & 0.463 \\
\hline Deep & $0.69 \pm 2.97$ & $1.00 \pm 3.64$ & $0.24 \pm 0.69$ & 0.559 \\
\hline Lobar & $0.41 \pm 1.15$ & $0.27 \pm 0.74$ & & 0.001 \\
\hline Lacunar infarctions & & & $4.10 \pm 3.71$ & 0.106 \\
\hline Total & $1.66 \pm 2.41$ & $2.10 \pm 1.81$ & $0.41 \pm 0.87$ & 0.001 \\
\hline Infratentorial & $0.17 \pm 0.76$ & $0.33 \pm 0.61$ & $3.03 \pm 2.96$ & 0.349 \\
\hline Deep & $1.03 \pm 1.80$ & $1.50 \pm 1.68$ & $0.66 \pm 1.14$ & \\
\hline Lobar & $0.45 \pm 0.95$ & $0.23 \pm 0.50$ & & 0.008 \\
\hline WM hyperintensities & & & $1.48 \pm 0.57$ & 0.016 \\
\hline Periventricular WM & $1.03 \pm 0.50$ & $1.23 \pm 0.43$ & $1.48 \pm 0.51$ & 0.042 \\
\hline Deep WM & $1.07 \pm 0.53$ & $1.33 \pm 0.55$ & $1.38 \pm 0.56$ & \\
\hline Total grade & $1.07 \pm 0.47$ & $1.17 \pm 0.38$ & & \\
\hline
\end{tabular}

Values are presented as mean \pm standard deviation.

baPWV, brachial-ankle pulse wave velocity; WM, white matter.

Table3: Multivariable analysis on the relationship between brachial-ankle pulse wave velocity and cerebral small vessel disease load

\begin{tabular}{|l|l|l|l|l|l|}
\hline & \multicolumn{2}{|l|}{ baPWV, Model 1 } & \multicolumn{2}{l|}{ baPWV, Model 2} \\
\hline & $\beta$ & $p$-value & \multicolumn{2}{l|}{} & \multicolumn{2}{l|}{$p$-value } \\
\hline Cerebral microbleeds & & & & & \\
\hline Total & 0.005 & 0.957 & & -0.001 & -0.990 \\
\hline Infratentorial & 0.093 & 0.270 & & 0.120 & 0.206 \\
\hline Deep & 0.000 & 1.000 & & -0.006 & 0.947 \\
\hline Lobar & -0.055 & 0.515 & & -0.078 & 0.413 \\
\hline Lacunar infarcts & & & & & \\
\hline Total & 0.226 & 0.007 & & 0.175 & 0.077 \\
\hline Infratentorial & -0.012 & 0.893 & & -0.017 & 0.856 \\
\hline Deep & 0.265 & 0.001 & & 0.304 & 0.001 \\
\hline Lobar & 0.181 & 0.035 & & 0.085 & 0.408 \\
\hline WM hyperintensities & & & & \\
\hline Periventricular WM & 0.179 & 0.044 & & 0.182 & 0.068 \\
\hline Deep WM & 0.117 & 0.203 & & 0.114 & 0.297 \\
\hline Total grade & 0.128 & 0.150 & & 0.099 & 0.332 \\
\hline
\end{tabular}

baPWV, brachial-ankle pulse wave velocity; WM, white matter. Values represent the standardized regression coefficients ( $\beta$ ). Model 1: Adjusted for age, sex, and systolic blood pressure. Model 2: adjusted for age, sex, systolic blood pressure, heart rate, hemoglobin level, erythrocyte sedimentation rate, and glomerular filtration rate.

\section{DISCUSSION}

This study is investigated the relationship between arterial stiffness and the imaging markers of CSVD in patients with acute ischemic stroke and found that increased baPWV was associated with the severity of deep lacunar infarction and deep and periventricular WMHs, but not with CMBs.However, in multivariate analysis, only deeplacunar infarction was independently associated with baPWV.

Arterial stiffness increases pulse pressure, 
leading to the development and progression of cardiovascular diseases.It is also a known independent predictor of CVD. ${ }^{3,11,12} \mathrm{Age}$, body weight, body mass index, total cholesterol level, low-density lipoprotein level, triglyceride level, fasting blood sugar level, and intima-media thickness of the internal carotid artery are known to be associated with baPWV. ${ }^{13,14}$ In acute ischemic stroke, the blood pressure rises due to cerebral blood flow autoregulation and stress, which may affect the arterial stiffnessduring the acute period.Although the biochemical changes affecting arterial stiffnessduring an acute ischemic stroke are not well known, arterial stiffness is believed to increase due to immuneinflammatory activation, vascular endothelial dysfunction, and other toxic effects. ${ }^{15}$ Arterial stiffnessmay have different measurement values even in the same patient due to daily blood pressure fluctuations. However,arterial stiffness isdetermined by several factors, including thestructural component of the arterial wall, tension in the vascular smooth muscle, and mean blood pressure. ${ }^{15}$ In this study, arterial stiffness was measured using baPWV, andin the acute stages of ischemic stroke, this can be affected by increased blood pressure due to stroke, size and locationof thestroke, and date of the test.However, arterial stiffness is not solely determined by high blood pressure.Especially in the acute period, where few antihypertensive drugs are used, it may be less responsiveto medication.Other previous studies conducted in the acute stage have not analyzed the effects of arterial stiffness on acute blood pressure changes due to the difficulty in correcting for or analyzing blood pressure, which continuously changes. All the previous studies included patients with ischemic stroke. As such, the effect of blood pressure may have been due to some effect in all patients. In this study, baPWV was associated with age, systolic and diastolic blood pressure, pulse pressure, heart rate, glomerular filtration rate, hemoglobin level, and erythrocyte sedimentation rate, but not with serum lipid level.

Arterial stiffness impairs the elasticity of the blood vessel walls, increases pulse pressure, and subsequently causessystemic hypertension. ${ }^{5}$ In particular, small arterioles in the brain have low vascular resistance, making them vulnerable to high pulsating systemic blood pressure, ${ }^{16,17}$ which may be damaging.Increased pulsatile stress disrupts the vascular endothelial cells and smooth muscles,causing blood vessel rupture.Systemic arterial stiffness affects earlier cerebral vessels more than other organs.Because CSVD is difficult to observe directly, brain imaging markers for CSVD are used. Although lacunar infarction, WMH, CMB, dilated PVS, and cerebral atrophy are imaging markersof CSVD, their pathophysiologies are different. Lacunar infarction and WMH are ischemic lesions caused by decreased perfusion, whileCMB is a hemorrhagic lesion. In contrast, dilated PVS is caused by the extravasation of interstitial fluids due to the increased permeability of theblood-brain barrier. As such,the relevant aspects of arterial stiffness and imaging markers may differ. ${ }^{18}$

A recent meta-analysis, showed that arterial stiffnesswas associated with lacunar infarction, $\mathrm{WMH}$, and CMB. ${ }^{6}$ Carotid-femoral PWV has been found to be associated with lacunar infarction, PVS of the basal ganglia, a wide range of WMHs, such as Fazeska 3. However, it wasnot associated with deepCMB, PVS of thecentral semiovale, and deep WMHs. ${ }^{8}$ It is notable that not all the imaging markers of CSVD areassociated with arterial stiffness.Based on the findings of the few studies on patients with acute ischemic stroke, baPWVis associated with chronic lacunar infarction, WMH, deep $\mathrm{CMB}$, and CSVD. ${ }^{7,15,19}$ Aortic PWV is associated with WMH volume and chronic lacunar infarction, ${ }^{20}$ but not with deepCMB. ${ }^{20,21}$ In one communitybased sample, PWV was related to PVS in the white matter, larger WMH volume, and lobar CMB.However, it was not associated withlacunar infarction. ${ }^{18}$ Studies have shown inconsistent results regarding the relationship between arterial stiffness and CMB..$^{6,7,18,20-22}$ In one study, PWV was associated with deepCMBs, but not with lobarCMBs. ${ }^{19}$ It is believed that the difference between the study group and the research method led to theinconsistent results. Unlike other studies, our study was conducted on patients with acute ischemic stroke. However, our results showed that chronic lacunar infarction and WMH were associated with baPWV, consistent with the results of previous studies. ${ }^{18}$ In particular, multivariate analysis, which adjusted for variables that may affect baPWV,showed that only deep lacunar infarction was significantly associated.CMB was not associated with baPWV, and no analysis of PVSor acute CSVD 
was performed.CMBmay be associated with elevated blood pressure, but it can also be caused by brain amyloid angiopathy. Therefore, mechanismsother than arterial stiffness may have affected it.This may not have reached statistical significance because of the low frequency of CMBs.CMBs and lacunar infarctionswere based on the number of lesions. Furthermore,WMH was analyzed by dividing thegrades according to severity, and since the number of study groups was small, it did not seem to be significant.

The limitations of this study are as follows. First, the study groupwas limited to patients with acute ischemic stroke, and there was no comparison with a normal control group. Second, the number of subjects was small, and other markers of CSVD, such as PVS, were not analyzed.There was also a lack of accurate assessment of peripheral vascular disease,leading to selection bias. Third, there was no analysis according to the mechanism of cerebral infarction, and there is a possibility that the acute cerebral infarction may have affected the variable. Fourth, the imaging evaluation was conducted by one person, and no inter-examiner reliability evaluation wasperformed.Prospective researchon more patientswill be neededin the future.

In conclusion, increased baPWV in patients with non-cardioembolic acute ischemic stroke was associated with lacunar infarction and WMH.Among the several markers of CSVD, deep lacunar infarction was a good marker of arterial stiffness. Systemic arterial stiffnessin some way affects CSVD, and baPWV may be valuable as a screening tool for CSVD.

\section{REFERENCES}

[1] Mancia G, De Backer G, Dominiczak A, Cifkova R, Fagard R, Germano G, et al. 2007 Guidelines for the management of arterial hypertension: the task force for the management of arterial hypertension of the European Society of Hypertension (ESH) and of the European Society of Cardiology (ESC). $J$ Hypertens 2007; 25:1105-1187.

[2] Yamashina A, Tomiyama H, Takeda K, Tsuda $\mathrm{H}$, Arai $\mathrm{T}$, Hirose $\mathrm{K}$, et al. Validity, reproducibility, and clinical significance of noninvasive brachial-ankle pulse wave velocity measurement. Hypertens Res 2002; 25:359364.

[3] Laurent S, Boutouyrie P, Asmar R, Gautier I, Laloux B, Guize L, et al. Aortic stiffness is an independent predictor of all-cause and cardiovascular mortality in hypertensive patients. Hypertension 2001; 37:1236-1241.

[4] O'Rourke MF, Staessen JA, Vlachopoulos C, Duprez D, Plante GE. Clinical applications of arterial stiffness; definitions and reference values.Am J Hypertens 2002; 15:426-444.

[5] Pantoni L. Cerebral small vessel disease: from pathogenesis and clinical characteristics to therapeutic challenges. Lancet Neurol 2010; 9:689-701.

[6] van Sloten TT, Protogerou AD, Henry RM, Schram MT, Launer LJ, Stehouwer CD. Association between arterial stiffness, cerebral small vessel disease and cognitive impairment: A systematic review and meta-analysis. NeurosciBiobehav Rev 2015; 53:121-130.

[7] Kim YB, Park KY, Chung PW, Kim JM, Moon HS, Youn YC. Brachial-ankle pulse wave velocity is associated with both acute and chronic cerebral small vessel disease. Atherosclerosis 2016; 245:54-59.

[8] Riba-Llena I, Jiménez-Balado J, CastañéX, Girona A, López-Rueda A, Mundet X, et al. Arterial stiffness is associated with basal ganglia enlarged perivascular spaces and cerebral small vessel disease load. Stroke 2018; 49:1279-1281.

[9] Gregoire SM, Chaudhary UJ, Brown MM, Yousry TA, Kallis C, Jäger HR, et al. The Microbleed Anatomical Rating Scale (MARS): reliability of a tool to map brain microbleeds. Neurology 2009; 73:1759-1766.

[10] Moon SY, Na DL, Seo SW, Lee JY, Ku BD, Kim SY, et al. Impact of white matter changes on activities of daily living in mild to moderate dementia. EurNeurol 2011; 65:223-230.

[11] Laurent S, Katsahian S, Fassot C, Tropeano AI, Gautier I, Laloux B, et al. Aortic stiffness is an independent predictor of fatal stroke in essential hypertension. Stroke 2003; 34:12031206.

[12] Mattace-Raso FU, van der Cammen TJ, Hofman A, van Popele NM, Bos ML, Schalekamp MA, et al. Arterial stiffness and risk of coronary heart disease and stroke: the Rotterdam Study. Circulation 2006; 113:657663.

[13] Munakata M, Ito N, Nunokawa T, Yoshinaga K. Utility of automated brachial ankle pulse wave velocity measurements in hypertensive patients. Am J Hypertens 2003; 16:653-657.

[14] Tomiyama H, Yamashina A, Arai T, Hirose K, Koji Y, Chikamori T, et al. Influences of age and gender on results of noninvasive brachialankle pulse wave velocity measurement--a survey of 12517 subjects. Atherosclerosis 2003; 166:303-309. 
[15] Tuttolomondo A, Di Sciacca R, Di Raimondo D, Serio A, D'Aguanno G, Pinto A, et al. Arterial stiffness indexes in acute ischemic stroke: relationship with stroke subtype.Atherosclerosis2010; 211:187-194.

[16] O’Rourke MF, Safar ME. Relationship between aortic stiffening and microvascular disease in brain and kidney: cause and logic of therapy. Hypertension 2005; 46:200-204.

[17] Faraci FM, Heistad DD. Regulation of large cerebral arteries and cerebral microvascular pressure. Circ Res 1990; 66:8-17.

[18] Zhai FF, Ye YC, Chen SY, Ding FM, Han F, Yang XL, et al. Arterial Stiffness and Cerebral Small Vessel Disease. Front Neurol 2018; 9:723.

[19] Song TJ, Kim J, Kim YD, Nam HS, Lee HS, Nam CM, et al. The distribution of cerebral microbleeds determines their association with arterial stiffness in non-cardioembolic acute stroke patients. Eur J Neurol 2014; 21:463469.

[20] Henskens LH, Kroon AA, van Oostenbrugge RJ, Gronenschild EH, Fuss-Lejeune MM, Hofman PA, et al. Increased aortic pulse wave velocity is associated with silent cerebral smallvessel disease in hypertensive patients. Hypertension 2008; 52:1120-1126.

[21] Poels MM, Zaccai K, Verwoert GC, Vernooij MW, Hofman A, van der Lugt A, et al. Arterial stiffness and cerebral small vessel disease: the rotterdam scan study. Stroke 2012; 43:26372642.

[22] Ochi N, Tabara Y, Igase M, Nagai T, Kido T, Miki $\mathrm{T}$, et al. Silent cerebral microbleeds associated with arterial stiffness in an apparently healthy subject. Hypertens Res $2009 ; 32: 255-260$.

Citation: MeyungKug Kim, Bong Goo Yoo. Association between Brachial-Ankle Arterial Stiffness and Cerebral Small Vessel Disease Load in Patients with Acute Non-Cardioembolic Ischemic Stroke. ARC Journal of Neuroscience. 2021; 6(1):1-7. doi: dx.doi.org/10.20431/2456-057X 0601001.

Copyright: (C) 2021 Authors. This is an open-access article distributed under the terms of the Creative Commons Attribution License, which permits unrestricted use, distribution, and reproduction in any medium, provided the original author and source are credited. 